\title{
Mindfulness Intervention Improves Cognitive Function in Older Adults by Enhancing the Level of miRNA-29c in Neuron-Derived Exosomes
}

Shin Hashizume

Sapporo Medical University

Masako Nakano ( $\nabla$ m.nakano@sapmed.ac.jp )

Sapporo Medical University

Kenta Kubota

Sapporo Medical University

Eiji Kobayashi

Sapporo Medical University

Mineko Fujimiya

Sapporo Medical University

\section{Research Article}

Keywords: MBSR, cognitive, MoCA-J, DNMT3A

Posted Date: February 17th, 2021

DOl: https://doi.org/10.21203/rs.3.rs-199928/v1

License: (9) This work is licensed under a Creative Commons Attribution 4.0 International License.

Read Full License 


\section{Abstract}

Although mindfulness-based stress reduction (MBSR) improves cognitive function, the mechanism is not clear. In this study, people aged 65 years and older were recruited from elderly communities in Chitose City, Japan, and assigned to the non-MBSR group or MBSR group. In the MBSR group, the program was performed three times a week for 4 weeks. After the intervention, the score of Japanese version of the Montreal Cognitive Assessment (MoCA-J) as well as the expression of microRNA (miR)-29c in neuronderived exosomes (NDEs) in blood was significantly increased in the MBSR group, compared to the nonMBSR group. Analysis of miR-29c target genes showed that the expression of DNA methyltransferase 3 alpha (DNMT3A), DNA methyltransferase 3 beta (DNMT3B), and signal transducer and activator of transcription 3 (STAT3) in NDEs was significantly decreased in the MBSR group. We also found that intracerebroventricular injection of a miR-29c mimic into 5xFAD mice prevented cognitive decline as well as neuronal loss in the subiculum area by down-regulating DNMT3A and STAT3 in the hippocampus. The results of the present study suggest that MBSR can prevent neuronal loss and cognitive impairment in elderly people by increasing the neuronal expression of miR-29c.

\section{Introduction}

Alzheimer's disease $(A D)$ is a progressive neurodegenerative disorder and is primarily responsible for dementia ${ }^{1}$. The pathological hallmarks of $A D$ are amyloid- $\beta(A \beta)$ plaques and neurofibrillary tangles in the brain ${ }^{2}$. MicroRNAs (miRNAs) were recently shown to be involved in $A \beta$ production, $A \beta$-induced neurotoxicity, and tau phosphorylation ${ }^{3}$. MiRNAs are non-coding RNAs 19-25 nucleotides in length that play a role in gene regulation by repressing the translation of target mRNAs ${ }^{4}$.

In the brains of AD patients, the expression of several miRNAs is dysregulated; miRNA (miR)-34a and miR-146a are up-regulated, whereas miRNAs miR-29c and miR-124 are down-regulated ${ }^{5}$. Because the dysregulated expression of miRNAs in the brain may reflect the circulating levels of miRNAs, the blood levels of miR-34a or miR-107 have been used as biomarkers for $A D^{6,7}$. However, circulating miRNAs are a mixture of those released from the brain and other tissues ${ }^{8}$. To detect miRNAs that originated from the brain, neuron-derived exosomes (NDEs) in the blood can be isolated using anti-L1 cell adhesion molecule antibody for $A D$ research ${ }^{9,10}$. Exosomes are 50 - to 100 -nm sized vesicles released from various kinds of cells and contain miRNA as well as mRNA, DNA, and proteins ${ }^{11}$. In AD patients, NDEs in the blood contain higher levels of $A \beta$ and tau, and lower levels of miR-212 compared to a control group ${ }^{9,10}$. However, whether these dysregulated molecules in NDEs could be normalized by treatment of AD is not known.

Treatments for $A D$ are divided into pharmacological and non-pharmacological interventions ${ }^{12,13}$. Among non-pharmacological interventions, evidence has shown that exercise can reduce the risk of developing dementia $^{14}$. In AD model mice, voluntary physical exercise can improve cognitive impairment by suppressing the expression of miR-132 in the hippocampus ${ }^{15}$. We previously reported that an enriched environment (EE) can prevent cognitive impairment in $A D$ and diabetes model animals by increasing the 
level of miR-146a in the brain and serum ${ }^{16,17}$. An EE is a housing condition that stimulates experiencedependent plasticity due to physical and social surroundings such as toys and mazes ${ }^{18}$. Although an EE contains an element of exercise, the effects of an EE are more effective for age-related cognitive impairment as well as anxiety conditions compared to physical exercise ${ }^{19}$. Although an EE cannot be applied directly to humans, exposure to mindfulness meditation as well as music lessons or physical activities may partially replicate an EE that has been studied in humans ${ }^{20}$.

Mindfulness-based stress reduction (MBSR) has emerged as a promising non-pharmacological intervention for $A D^{21}$. MBSR is a meditation in which the person pays attention and is aware of his or her ongoing experience in the present moment ${ }^{22}$. A program of MBSR that was designed by Jon Kabat-Zinn in 1979 has shown much evidence for reducing chronic pain, depression, and anxiety ${ }^{23-25}$. The mechanism of MBSR for psychological improvement involves the regulation of activity in brain regions including the amygdala, anterior cingulate cortex, and dorsolateral prefrontal cortex ${ }^{26}$. The effectiveness of MBSR for mild cognitive impairment $(\mathrm{MCl})$ and $A D$ has been shown in several studies over the last 10 years ${ }^{27-30}$. A randomized study of 120 AD patients in 2016 confirmed the effectiveness of MBSR for maintaining cognitive function ${ }^{31}$.

Although MBSR increases cerebral blood flow and reduces blood inflammatory proteins in older adults with memory loss ${ }^{27,32}$, no previous studies have shown whether levels of miRNA in the brain are changed by MBSR. Therefore, the purpose of this study is to elucidate the mechanism of effectiveness of MBSR on cognitive function by focusing on miRNAs in NDEs.

\section{Methods}

\section{Participants}

This study was approved by the Sapporo Medical University ethics committee (approval number 30-2-17). All methods were carried out according to the principles of the Declaration of Helsinki. Participants aged 65 years and older were recruited from three elderly communities in Chitose City, Japan. After providing an explanation of the study to the participants, we obtained written informed consent. We excluded participants with severe hypertension, heart disease, psychiatric disorders, and upper and lower limb motor difficulties.

\section{Procedure and intervention}

A total of 45 older adults were participated in this study. Among the three communities, participants in two communities were assigned to Group $1(n=20)$, and those in the third community were assigned to Group $2(\mathrm{n}=25)$. The study flow and participant groups are shown in Fig. 1. We surveyed participants' age, sex, height, weight, exercise habits (frequency of exercise per week and duration of exercise per session), years of education, history of smoking, and number of drinks prior to the intervention. In Group 1, no intervention was performed for 4 weeks, and then MBSR was performed for 4 weeks. In Group 2, 
only the intervention of MBSR was performed for 4 weeks (Fig. 1a). During the intervention, three MBSR sessions were performed per week, for a total of 12 sessions within 4 weeks. Participants performed a body scan (1st week), yoga meditation (2nd week), sitting meditation (3rd week), and yoga meditation (4th week). We used the CDs of the Japanese translation of the book of "Guided Mindfulness Meditation" that was written by Jon Kabat-Zinn ${ }^{33}$. The sessions were performed for 60 min each time, and we excluded yoga postures that are difficult for elderly people. Before and after the intervention, we assessed cognitive function using the Japanese version of the Montreal Cognitive Assessment (MoCA-J) and collected blood samples. We excluded the data of participants who performed the MBSR program fewer than eight times or who did not undergo the second or third assessment. Finally, we obtained the data for nine people in the non-MBSR group and 22 people in the MBSR group (Fig. 1b).

\section{Blood collection, isolation of NDEs and quantitation of miRNAs as well as mRNA}

Blood samples were collected from participants using a vacuum blood collection tube, and plasma was collected $24 \mathrm{~h}$ later by centrifugation at $4{ }^{\circ} \mathrm{C}$ at $300 \times \mathrm{g}$ for $10 \mathrm{~min}$ and stored at $-80^{\circ} \mathrm{C}$. Isolation of NDEs from total exosomes was performed according a previous study ${ }^{9}$. Targeted miRNA and mRNA that listed in Supplementary Table 1 and Supplementary Table 2 were measured. The detailed methods are described in Supplementary information.

\section{Animals}

We performed animal experiments in accordance with the approved guidelines of the Animal Experiment Committee of Sapporo Medical University (Sapporo, Japan). All experimental protocols were approved by the Animal Experiment Committee of Sapporo Medical University (approval \#19-052). The study was carried out in compliance with the ARRIVE guidelines. 5xFAD mice were maintained at a temperature of $21-24{ }^{\circ} \mathrm{C}$ and humidity of $50-70 \%$, with a 12 -h light/12-h dark cycle. Food and water were available ad libitum.

We made every effort to eliminate pain and distress in the animals. We used isoflurane inhalation when invasive procedures were needed, and confirmed the absence of the toe pinch reflex before performing the procedures. At the end of the experiments, excess isoflurane inhalation was carried out to euthanize the animals. After confirming cardiopulmonary arrest, all blood was collected from the heart of the animals.

\section{Animal experiment}

To investigate whether injection of the miR-29c mimic prevents a decline in cognitive function, we injected negative control miRNA or miR-29c mimic (140 pmol/animal) (Thermo Fisher Scientific, Waltham, MA, USA) into 5-month-old 5xFAD mice (\#034848, The Jackson Laboratory, Bar Harbor, ME, USA) four times at 1-week intervals. At 1 week after the last injection, $Y$ maze tests were conducted, and mice were sacrificed at 6 months old for further analysis. Excess isoflurane inhalation was carried out to euthanize the mice. After all blood was collected, brains were removed from the skull and divided into the 
left and right hemispheres with a scalpel. The right hemisphere was used for PCR analysis, and the left hemisphere was used for immunohistochemical analysis. The primers of miRNA and mRNA are listed in Supplementary Table 1 and Supplementary Table 3. The antibodies used for immunohistochemical analysis are shown in Supplementary Table 4. The detailed methods are described in Supplementary information.

\section{Statistical analysis}

Data were expressed as the mean \pm standard deviation (SD) or standard error of the mean (SEM). Statistical analysis was performed using the paired or unpaired $t$-test ( $R$ version 3.6.1). The data of the MoCA-J was also analyzed with two-way repeated measures analysis of variance (ANOVA).

\section{Results}

\section{Baseline demographics and characteristics}

Table 1 shows the demographics of each group before the MBSR intervention including age, sex, education level, exercise habit, and the MoCA-J score. No significant differences were observed between the non-MBSR and MBSR groups in any items.

\section{MBSR improved cognitive function as assessed by MoCA-J.}

The MoCA-J tests were performed to evaluate cognitive function before and after the intervention in the non-MBSR and MBSR groups. The total score for the MoCA-J did not change in the non-MBSR group (Fig. 2a), whereas the score was significantly increased in the MBSR group after the intervention (Fig. 2b). Among the seven domains of the MoCA-J, the score for visuospatial/executive function was significantly decreased in the non-MBSR group (Fig. 3a). On the other hand, the scores for delayed recall, visuospatial/executive function, and attention were significantly increased in the MBSR group (Fig. 3b). The effects of MBSR against visuospatial/executive function as well as attention were also confirmed by two-way repeated ANOVA (Supplementary Table 5).

\section{MBSR enhanced the expression of miR-29c in NDEs.}

Next, we analyzed the expression of miRNAs in exosomes derived from serum in both groups. The expression of miRNAs related to AD pathogenesis including miR-9, miR-29c, miR-124, miR-125b, miR$146 \mathrm{a}$, and miR-181a was analyzed. No significant changes were found in any measured miRNA in either group (Supplementary Table 6). Therefore, we isolated NDEs from serum-derived exosomes and analyzed the expression of miRNAs in NDEs in both groups. No significant changes were found in any measured miRNAs in NDEs in the non-MBSR group. On the other hand, the expression of miR-29c in NDEs was significantly increased in the MBSR group after the intervention (Table 2).

\section{MBSR suppressed the expression of DNMT3A, DNMT3B, and STAT3 in NDEs.}


Next, we examined target genes that may be affected by the increase in miR-29c. We analyzed the expression of DNA methyltransferase 3 alpha (DNMT3A), DNA methyltransferase 3 beta (DNMT3B), signal transducer and activator of transcription 3 (STAT3), and beta-site amyloid precursor protein cleaving enzyme 1 (BACE1) in NDEs. We found no significant changes in any measured mRNAs in the non-MBSR group. On the other hand, we found significant decreases in the expression of DNMT3A, DNMT3B, and STAT3 in the MBSR group after the intervention (Table 3).

\section{Intracerebroventricular injection of miR-29c mimic prevented cognitive impairment in 5xFAD mice.}

To investigate the type of molecular and pathological changes that occur due to the increase in miR-29c in the central nervous system (CNS), we injected the miR-29c mimic intracerebroventricularly into $A D$ model mice. We used 5xFAD mice that have three mutations in amyloid precursor protein (APP) and two mutations in presenilin-1 (PSEN1) ${ }^{34}$. 5xFAD mice show no cognitive impairment at 5 months old, but exhibit cognitive decline beginning at 6 months old (Supplementary Fig. 1a and 1b). To investigate whether injection of the miR-29c mimic prevents a decline in cognitive function, we injected negative control miRNA or miR-29c mimic (140 pmol/animal) into 5-month-old 5xFAD mice four times at 1-week intervals. At 1 week after the last injection, $Y$ maze tests were conducted, and mice were sacrificed at 6 months old for further analysis (Fig. 4a).

In the $Y$ maze tests, the total number of arm entries was not different between the two groups. However, the percentage of alternation, which represents cognitive function, was significantly higher in the miR-29c mimic group than the negative control group (Fig. 4b).

\section{The increase in miR-29c in the CNS does not affect the level of $A \beta$, but prevents the decrease in neuronal number in the hippocampus by down-regulating the level of DNMT3A and STAT3.}

We investigated the mechanisms by which the miR-29c mimic prevents cognitive impairment in 5xFAD mice. The hippocampal expression of miR-29c was significantly increased in the miR-29c mimic-injected group compared to the negative control group. On the other hand, the hippocampal expression of DNMT3A, STAT3, and BACE1 was significantly decreased in the miR-29c mimic-injected group compared to the negative control group (Fig. 4c).

Because BACE1 is the enzyme that cleaves APP at the $\beta$-site and produces $A \beta^{35}$, we evaluated the expression of $A \beta$ in the subiculum area. However, we found no significant differences in the positive area of $A \beta$ between the groups (Fig. $4 \mathrm{~d}$ ).

Because decreased expression of DNMT3A and STAT3 inhibits neuronal apoptosis ${ }^{36,37}$, we evaluated the number of NeuN-positive cells in the subiculum area. The number of NeuN-positive cells was significantly higher in the miR-29c mimic-injected group than the negative control group (Fig. 4e).

\section{Discussion}


To the best of our knowledge, this is the first report to reveal the mechanism behind the effect of MBSR on cognitive function by focusing on miRNA. To evaluate how MBSR changes the expression of miRNAs in the brain, we examined NDEs isolated from blood. We revealed that MBSR not only improved cognitive function in older adults but also increased the level of miR-29c in NDEs. In addition, we found that the expression of DNMT3A, DNMT3B, and STAT3, which are target genes of miR-29c, was decreased in NDEs after MBSR. Furthermore, we found that intracerebroventricular injection of miR-29c improved cognitive function and inhibited neuronal apoptosis in 5XFAD mice by down-regulating the expression of DNMT3A and STAT3 in the hippocampus. Therefore, MBSR may have an effect on preserving neuronal number by increasing the expression of miR-29c in the CNS.

MBSR improves the cognitive function in $\mathrm{MCl}$ and $A D$ patients ${ }^{21,27,29-31}$. Although MBSR is typically an 8week program, we performed MBSR for 4 weeks because this shortened version of MBSR is fully effective for psychological problems including stress ${ }^{38,39}$. Jon Kabat-Zinn recommends a program of 8 weeks that consists of a body scan (1-2 weeks), the combination of a body scan and yoga meditation (3-4 weeks), the combination of yoga and sitting meditation (5- 6 weeks), and a free combination of a body scan, yoga, or sitting meditation (7-8 weeks) ${ }^{40}$. A body scan involves focusing on the sensations in parts of body in a gradual sequence from feet to head ${ }^{40}$. In this study, we shortened this 8-week program into a 4week program that consists of a body scan (1st week), yoga meditation (2nd week), sitting meditation (3rd week), and yoga meditation (4th week).

To evaluate cognitive function, we used the Japanese version of the MoCA that was originally written in English by Nasreddine et al. in 2005 41,42 . The MoCA was developed as a brief screening test for $\mathrm{MCl}$ to address the limitations of the Mini-Mental State Examination ${ }^{42}$. In the present study, the MoCA-J score of all participants at baseline was $22.1 \pm 3.1$ (mean $\pm S D$ ). Rossetti et al. reported that the MoCA score of people of different races aged $70-80$ years was $21.32 \pm 4.78$ (mean \pm SD) ${ }^{43}$; the MoCA-J score in Japanese people aged $85-87$ years was $22.9 \pm 3.5$ (mean \pm SD) ${ }^{44}$. Considering that the age of the participants in this study was $78.9 \pm 5.5$ years (mean \pm SD), their cognitive function did not seem to be significantly different from that reported in previous studies.

The total score for the MoCA-J did not change in the non-MBSR group, although the score did significantly increase in the MBSR group after the intervention $(P=1.863 \mathrm{e}-06)$. For the diagnosis of $\mathrm{MCl}$, a score $\leq 25$ is the optimal cut-off point in the MoCA test ${ }^{45}$. In the present study, among 22 participants in the MBSR group, 19 participants showed a score of 25 or less before the MBSR intervention. When excluding the three cognitively normal people, the 19 participants also showed an improvement in cognitive function following MBSR $(P=2.903 \mathrm{e}-06)$ (Supplementary Fig. 2). Thus, similar to a previous study $^{29}$, we found that MBSR was effective for improving cognitive impairment in older people with $\mathrm{MCl}$.

In the detailed analysis of the MoCA-J results, the scores for visuospatial/executive functions, delayed recall, and attention significantly improved after the MBSR intervention compared to before the MBSR intervention. Brief meditation training improves visuospatial processing and executive functioning ${ }^{46}$. In 
addition, MBSR is effective for delayed recall as well as verbal learning ${ }^{30,47}$. Furthermore, brief mindfulness meditation improves attention, even in novices ${ }^{48}$. Therefore, our results were consistent with previous studies. In the non-MBSR group, the scores for visuospatial/executive functions significantly decreased after the non-intervention. We do not know the reason, but executive function is frequently impaired in $\mathrm{MCl}$ because the integrity of the white matter in frontal and cingulate regions is dysregulated ${ }^{49}$.

We found that the expression of miR-29c in NDEs was increased in the MBSR group. Both cerebral expression of miR-29c and its level in blood are decreased in AD patients ${ }^{50,51}$. Because the expression of miR-29c in NDEs was up-regulated by MBSR in the present study, the neuronal expression of miR-29c in the CNS is likely to be increased.

Because miR-29c suppresses the target genes of DNMT3A, DNMT3B, STAT3, and BACE1 ${ }^{36,50,51}$, we analyzed the expression of these genes in NDEs. We found that the MBSR intervention decreased the expression of DNMT3A, DNMT3B, and STAT3. DNMT3A and DNMT3B are the main DNA methyltransferases that initiate DNA methylation in neurons ${ }^{52}$. DNA methylation is a crucial epigenetic mark for the regulation of gene transcription ${ }^{53}$. The level of miR-29c is decreased in a rat ischemic brain damage model, and treatment with miR-29c inhibits neuronal cell death by suppressing DNMT3A ${ }^{36}$. Because the expression of miR-29c was increased and DNMT3A was decreased in NDEs in this study, neuronal death is likely to be inhibited by MBSR. Additionally, DNMT3A and DNMT3B are increased in 5XFAD model mice ${ }^{54}$, and higher cytosine methylation is related to cognitive impairment ${ }^{54}$. Because the MBSR intervention decreased the expression of DNMT3A and DNMT3B in NDEs, these molecular changes may be involved in the up-regulation of gene transcription that promotes cognitive function. In addition, STAT3 mediates A $\beta$-induced neuronal cell death ${ }^{37}$. Because decreased expression of STAT3 was observed in NDEs, MBSR may inhibit neuronal death. We also evaluated the expression of BACE1 in this study, but no significant change was observed following MBSR. Because BACE1 is an enzyme that cleaves APP and produces $A \beta^{35}$, MBSR may not affect $A \beta$ levels.

In the present study, the expression of miRNAs including miR-9, miR-124, miR-125b, miR-146a and miR181a in NDEs was not changed by MBSR. MiR-9 and miR-124 play a role in repression of BACE1, and both miRNAs are down-regulated in the brain of $A D$ patients ${ }^{5}$. On the other hand, the expression of miR$125 \mathrm{~b}$ is up-regulated in the brains of $A D$ patients, and phosphorylation of tau is induced by miR-125b55. Because no significant changes were observed in miR-9, miR-124, or miR-125b, MBSR is assumed not to reduce the production of $A \beta$ or suppress the phosphorylation of tau. MiR-146a has an anti-inflammatory effect by suppressing the expression of NF-KB; however, miR-146a is increased in the brains of $A D$ patients ${ }^{56}$. We previously reported that intracerebroventricular injection of mesenchymal stem cells improves cognitive impairment in AD model mice by transferring exosomal miR-146a into the $\mathrm{CNS}^{17}$. In the present study, we found no significant change in the expression of miR-146a in NDEs, suggesting that miR-146a may not be involved in the cognitive improvement by MBSR. MiR-181a enhances hippocampus-dependent memory by targeting protein kinase AMP-activated catalytic subunit alpha 1 
(PRKAA1) ${ }^{57}$. Because no difference was found in the expression of miR-181a, MBSR may not affect the expression of PRKAA1.

To investigate why neuronal up-regulation of miR-29c by MBSR is related to cognitive improvement, we injected miR-29c mimic intracerebroventricularly into 5xFAD mice. The $Y$ maze test was then conducted to assess short-term memory ${ }^{58}$. We found that the up-regulation of miR-29c prevented cognitive impairment in 5xFAD mice possibly by inhibiting the expression of DNMT3A, STAT3, and BACE1 in the hippocampus. A discrepancy was found in the result of BACE1 between humans and mice; the expression of BACE1 in NDEs was not changed in the MBSR group. However, the decrease in BACE1 in mice may not have influenced the production of $A \beta$, because the $A \beta$-positive area in the subiculum was not changed by up-regulation of miR-29c. Because $5 x F A D$ is a model that has five mutations and rapidly develops severe amyloid pathology, decreased expression of BACE1 may not be sufficient to reduce $A \beta$. On the other hand, the number of NeuN-positive cells was significantly higher in the miR-29c mimicinjected group. Because down-regulation of DNMT3A and STAT3 inhibits neuronal apoptosis ${ }^{36,37}$, these molecular changes may have a positive effect on preservation of neuronal number. We previously reported that preservation of neuronal number is associated with normal cognitive function in human subjects with severe accumulation of $A \beta$ and tau in the brain ${ }^{59}$. Therefore, preservation of neuronal number in the miR-29c mimic-injected group may have contributed to the prevention of cognitive decline, despite no decline in A $\beta$. Thus, MBSR may have an effect in inhibiting neuronal apoptosis by upregulating miR-29c in neurons.

This is the first study to show that MBSR improves cognitive function by up-regulating the expression of miR-29c in NDEs. Among non-pharmacological interventions, exercise is a well-known method to reduce the risk of $A D^{14}$. Exercise increases the level of miR-21 in serum ${ }^{60}$ and enhances epigenetic changes including DNA methylation in the $\mathrm{CNS}^{61}$. Although MBSR involves slight body movement, the average energy expenditure during yoga and meditation are lower than during walking ${ }^{62}$. Thus, the physical element of MBSR may not have contributed to the change in miR-29c in NDEs.

Although we found a new mechanism regarding the effectiveness of MBSR on cognitive function, we did not identify how epigenetic changes were induced by the decrease in DNMT3A and DNMT3B. The increase in DNA methylation of the brain-derived neurotrophic factor (BDNF) promoter may be responsible for the reduction in BDNF mRNA or protein in $A D$ patients ${ }^{63,64}$. In addition, enhanced DNA methylation accompanied by an increase in DNMT3 is related to a decrease in miR-29c in AD patients ${ }^{64}$. Therefore, the up-regulation of miR-29c induced by MBSR may have enhanced the transcription of BDNF by down-regulating DNMT3A and DNMT3B.

In summary, MBSR improved cognitive function in older adults possibly by increasing neuronal expression of miR-29c. Mesenchymal stem cell-derived exosomes containing miR-29b and miR-146a are effective in AD models by transferring the exosomes into the CNS ${ }^{65,66}$. However, we found that MBSR can increase the neuronal expression of miR-29c without the injection of stem cells or exosomes. These 
finding suggest that mindfulness can prevent the onset of dementia and regulate miRNAs in the body, even if pharmacological interventions are not included. Further evidence is expected for the establishment of MBSR as a method for preventing or treating cognitive impairment.

\section{Declarations}

\section{Acknowledgments}

This research was supported by funding from JSPS KAKENHI Grant Number JP19K16985 and LEOC Co., Ltd.

\section{Author contributions}

S.H., M.N., K.K. and M.F. designed the study, analyzed the data, and wrote the paper. S.H. did the program of MBSR. S.H., M.N., K.K. did the 1st, 2nd, and 3rd assessments. E.K. contributed to the interpretation of data.

\section{Additional Information}

All authors declare no conflict of interest.

\section{References}

1. Cornutiu, G. The Epidemiological Scale of Alzheimer's Disease. J Clin Med Res. 7, 657-666 (2015).

2. Bloom, G. S. Amyloid- $\beta$ and tau: the trigger and bullet in Alzheimer disease pathogenesis. JAMA Neurol. 71, 505-508 (2014).

3. Wang, M., Qin, L. \& Tang, B. MicroRNAs in Alzheimer's Disease. Front Genet. 10, 153 (2019).

4. Lu, T. X., Rothenberg, M. E. \& MicroRNA J Allergy Clin Immunol. 141, 1202-1207 (2018).

5. Miya Shaik, M. et al. The Role of microRNAs in Alzheimer's Disease and Their Therapeutic Potentials.Genes9 (2018).

6. Kumar, S. \& Reddy, P. H. Are circulating microRNAs peripheral biomarkers for Alzheimer's disease? Biochim Biophys Acta. 1862, 1617-1627 (2016).

7. Swarbrick, S., Wragg, N., Ghosh, S. \& Stolzing, A. Systematic Review of miRNA as Biomarkers in Alzheimer's Disease. Mol Neurobiol. 56, 6156-6167 (2019).

8. Wang, Z., Lu, Y. \& Han, J. Peripheral blood microRNAs: A novel tool for diagnosing disease? Intractable Rare Dis Res. 1, 98-102 (2012).

9. Winston, C. N. et al. Prediction of conversion from mild cognitive impairment to dementia with neuronally derived blood exosome protein profile. Alzheimers Dement. 3, 63-72 (2016).

10. Cha, D. J. et al. miR-212 and miR-132 Are Downregulated in Neurally Derived Plasma Exosomes of Alzheimer's Patients. Front Neurosci. 13, 1208 (2019). 
11. Barile, L., Vassalli, G. \& Exosomes Therapy delivery tools and biomarkers of diseases. Pharmacol Ther. 174, 63-78 (2017).

12. Epperly, T., Dunay, M. A. \& Boice, J. L. Alzheimer Disease: Pharmacologic and Nonpharmacologic Therapies for Cognitive and Functional Symptoms. Am Fam Physician. 95, 771-778 (2017).

13. Olazarán, J. et al. Nonpharmacological therapies in Alzheimer's disease: a systematic review of efficacy. Dement Geriatr Cogn Disord. 30, 161-178 (2010).

14. Ridler, C. Exercise wards off Alzheimer disease by boosting neurogenesis and neuroprotective factors. Nat Rev Neurol. 14, 632 (2018).

15. Dong, J., Liu, Y., Zhan, Z. \& Wang, X. MicroRNA-132 is associated with the cognition improvement following voluntary exercise in SAMP8 mice. Brain Res Bull. 140, 80-87 (2018).

16. Kubota, K. et al. An enriched environment prevents diabetes-induced cognitive impairment in rats by enhancing exosomal miR-146a secretion from endogenous bone marrow-derived mesenchymal stem cells. PLoS One. 13, e0204252 (2018).

17. Nakano, M. et al. An enriched environment prevents cognitive impairment in an Alzheimer's disease model by enhancing the secretion of exosomal microRNA-146a from the choroid plexus. Brain, Behavior, \& Immunity - Health 9, 100149(2020).

18. Nithianantharajah, J. \& Hannan, A. J. Enriched environments, experience-dependent plasticity and disorders of the nervous system. Nat Rev Neurosci. 7, 697-709 (2006).

19. Singhal, G. et al. Short-term environmental enrichment, and not physical exercise, alleviate cognitive decline and anxiety from middle age onwards without affecting hippocampal gene expression. Cogn Affect Behav Neurosci. 19, 1143-1169 (2019).

20. McCreary, J. K. \& Metz, G. A. S. Environmental enrichment as an intervention for adverse health outcomes of prenatal stress. Environ Epigenet. 2, dvw013 (2016).

21. Russell-Williams, J. et al. Mindfulness and meditation: treating cognitive impairment and reducing stress in dementia. Rev Neurosci. 29, 791-804 (2018).

22. Creswell, J. D. Mindfulness Interventions. Annu Rev Psychol. 68, 491-516 (2017).

23. Kabat-Zinn, J. An outpatient program in behavioral medicine for chronic pain patients based on the practice of mindfulness meditation: theoretical considerations and preliminary results. Gen Hosp Psychiatry. 4, 33-47 (1982).

24. Parmentier, F. B. R. et al. Mindfulness and Symptoms of Depression and Anxiety in the General Population: The Mediating Roles of Worry, Rumination, Reappraisal and Suppression. Front Psychol. 10, 506 (2019).

25. Kabat-Zinn, J. et al. Effectiveness of a meditation-based stress reduction program in the treatment of anxiety disorders. Am J Psychiatry. 149, 936-943 (1992).

26. Tang, Y. Y., Hölzel, B. K. \& Posner M. I. The neuroscience of mindfulness meditation. Nat Rev Neurosci. 16, 213-225 (2015). 
27. Newberg, A. B., Wintering, N., Khalsa, D. S., Roggenkamp, H. \& Waldman, M. R. Meditation effects on cognitive function and cerebral blood flow in subjects with memory loss: a preliminary study. $J$ Alzheimers Dis. 20, 517-526 (2010).

28. Smart, C. M., Segalowitz, S. J., Mulligan, B. P., Koudys, J. \& Gawryluk, J. R. Mindfulness Training for Older Adults with Subjective Cognitive Decline: Results from a Pilot Randomized Controlled Trial. $J$ Alzheimers Dis. 52, 757-774 (2016).

29. Wong, W. P., Coles, J., Chambers, R., Wu, D. B. \& Hassed, C. The Effects of Mindfulness on Older Adults with Mild Cognitive Impairment. J Alzheimers Dis Rep. 1, 181-193 (2017).

30. Lenze, E. J. et al. Mindfulness-based stress reduction for older adults with worry symptoms and cooccurring cognitive dysfunction. Int J Geriatr Psychiatry. 29, 991-1000 (2014).

31. Quintana-Hernández, D. J. et al. Mindfulness in the Maintenance of Cognitive Capacities in Alzheimer's Disease: A Randomized Clinical Trial. J Alzheimers Dis. 50, 217-232 (2016).

32. Ng, T. K. S. et al. Mindfulness improves inflammatory biomarker levels in older adults with mild cognitive impairment: a randomized controlled trial. Transl Psychiatry. 10, 21 (2020).

33. Kabat-Zinn, J. Guided Mindfulness Meditation(SOUNDS TRUE INC, 2005).

34. Oakley, H. et al. Intraneuronal beta-amyloid aggregates, neurodegeneration, and neuron loss in transgenic mice with five familial Alzheimer's disease mutations: potential factors in amyloid plaque formation. J Neurosci. 26, 10129-10140 (2006).

35. Schubert, C. Alzheimer disease: BACE1 branches out. Nat Med. 12, 1123 (2006).

36. Pandi, G., Nakka, V. P., Dharap, A., Roopra, A. \& Vemuganti, R. MicroRNA miR-29c down-regulation leading to de-repression of its target DNA methyltransferase 3a promotes ischemic brain damage. PLoS One. 8, e58039 (2013).

37. Wan, J. et al. Tyk2/STAT3 signaling mediates beta-amyloid-induced neuronal cell death: implications in Alzheimer's disease. J Neurosci. 30, 6873-6881 (2010).

38. Demarzo, M. et al. Efficacy of 8- and 4-Session Mindfulness-Based Interventions in a Non-clinical Population: A Controlled Study. Front Psychol. 8, 1343 (2017).

39. Braden, B. B. et al. Brain and behavior changes associated with an abbreviated 4-week mindfulnessbased stress reduction course in back pain patients. Brain Behav. 6, e00443 (2016).

40. Kabat-Zinn, J. Full Catastrophe Living (Revised Edition)(Random House, 2013).

41. Fujiwara, Y. et al. Brief screening tool for mild cognitive impairment in older Japanese: validation of the Japanese version of the Montreal Cognitive Assessment. Geriatr Gerontol Int. 10, 225-232 (2010).

42. Nasreddine, Z. S. et al. The Montreal Cognitive Assessment, MoCA: a brief screening tool for mild cognitive impairment. J Am Geriatr Soc. 53, 695-699 (2005).

43. Rossetti, H. C., Lacritz, L. H., Cullum, C. M. \& Weiner, M. F. Normative data for the Montreal Cognitive Assessment (MoCA) in a population-based sample. Neurology. 77, 1272-1275 (2011). 
44. Godai, K. et al. Day-to-day blood pressure variability is associated with lower cognitive performance among the Japanese community-dwelling oldest-old population: the SONIC study. Hypertens Res. 43, 404-411 (2020).

45. Sobreira, E. et al. Screening of cognitive impairment in patients with Parkinson's disease: diagnostic validity of the Brazilian versions of the Montreal Cognitive Assessment and the Addenbrooke's Cognitive Examination-Revised. Arq Neuropsiquiatr. 73, 929-933 (2015).

46. Zeidan, F., Johnson, S. K., Diamond, B. J., David, Z. \& Goolkasian, P. Mindfulness meditation improves cognition: evidence of brief mental training. Conscious Cogn. 19, 597-605 (2010).

47. Lueke, A. \& Lueke, N. Mindfulness improves verbal learning and memory through enhanced encoding. Mem Cognit. 47, 1531-1545 (2019).

48. Norris, C. J., Creem, D., Hendler, R. \& Kober, H. Brief Mindfulness Meditation Improves Attention in Novices: Evidence From ERPs and Moderation by Neuroticism. Front Hum Neurosci. 12, 315 (2018).

49. Reinvang, I., Grambaite, R. \& Espeseth, T. Executive Dysfunction in MCl: Subtype or Early Symptom. Int J Alzheimers Dis 2012, 936272 (2012).

50. Lei, X., Lei, L., Zhang, Z., Zhang, Z. \& Cheng, Y. Downregulated miR-29c correlates with increased BACE1 expression in sporadic Alzheimer's disease. Int J Clin Exp Pathol. 8, 1565-1574 (2015).

51. Wu, Y. et al. Lower Serum Levels of miR-29c-3p and miR-19b-3p as Biomarkers for Alzheimer's Disease. Tohoku J Exp Med. 242, 129-136 (2017).

52. Bayraktar, G., Kreutz, M. R., Neuronal, D. N. A. \& Methyltransferases Epigenetic Mediators between Synaptic Activity and Gene Expression? Neuroscientist. 24, 171-185 (2018).

53. Suzuki, M. M. \& Bird, A. DNA methylation landscapes: provocative insights from epigenomics. Nature Reviews Genetics. 9, 465-476 (2008).

54. Griñán-Ferré, C. et al. Epigenetic mechanisms underlying cognitive impairment and Alzheimer disease hallmarks in 5XFAD mice. Aging. 8, 664-684 (2016).

55. Ma, X., Liu, L. \& Meng, J. MicroRNA-125b promotes neurons cell apoptosis and Tau phosphorylation in Alzheimer's disease. Neurosci Lett. 661, 57-62 (2017).

56. Alexandrov, P. N., Dua, P. \& Lukiw, W. J. Up-Regulation of miRNA-146a in Progressive, Age-Related Inflammatory Neurodegenerative Disorders of the Human CNS. Front Neurol. 5, 181 (2014).

57. Zhang, S. F., Chen, J. C., Zhang, J. \& Xu, J. G. miR-181 a involves in the hippocampus-dependent memory formation via targeting PRKAA1. Sci Rep. 7, 8480 (2017).

58. Kraeuter, A. K., Guest, P. C. \& Sarnyai, Z. The Y-Maze for Assessment of Spatial Working and Reference Memory in Mice. Methods Mol Biol. 1916, 105-111 (2019).

59. Kobayashi, E. et al. Activated forms of astrocytes with higher GLT-1 expression are associated with cognitive normal subjects with Alzheimer pathology in human brain. Sci Rep. 8, 1712 (2018).

60. Zhou, Q. et al. Circulating microRNAs in Response to Exercise Training in Healthy Adults. Front Genet. 11,256 (2020). 
61. Fernandes, J., Arida, R. M. \& Gomez-Pinilla, F. Physical exercise as an epigenetic modulator of brain plasticity and cognition. Neurosci Biobehav Rev. 80, 443-456 (2017).

62. Ray, U. S., Pathak, A. \& Tomer, O. S. Hatha yoga practices: energy expenditure, respiratory changes and intensity of exercise. Evid Based Complement Alternat Med 2011, 241294 (2011).

63. Nagata, T. et al. Association between DNA Methylation of the BDNF Promoter Region and Clinical Presentation in Alzheimer's Disease. Dement Geriatr Cogn Dis Extra. 5, 64-73 (2015).

64. Yang, G. et al. DNA methyltransferase 3, a target of microRNA-29c, contributes to neuronal proliferation by regulating the expression of brain-derived neurotrophic factor. Mol Med Rep. 12, 1435-1442 (2015).

65. Jahangard, Y. et al. Therapeutic Effects of Transplanted Exosomes Containing miR-29b to a Rat Model of Alzheimer's Disease. Front Neurosci. 14, 564 (2020).

66. Nakano, M. et al. Bone marrow-derived mesenchymal stem cells improve cognitive impairment in an Alzheimer's disease model by increasing the expression of microRNA-146a in hippocampus. Sci Rep. 10, 10772 (2020).

\section{Tables}

Due to technical limitations, table 1,2,3 is only available as a download in the Supplemental Files section.

\section{Figures}


Figure 1

a

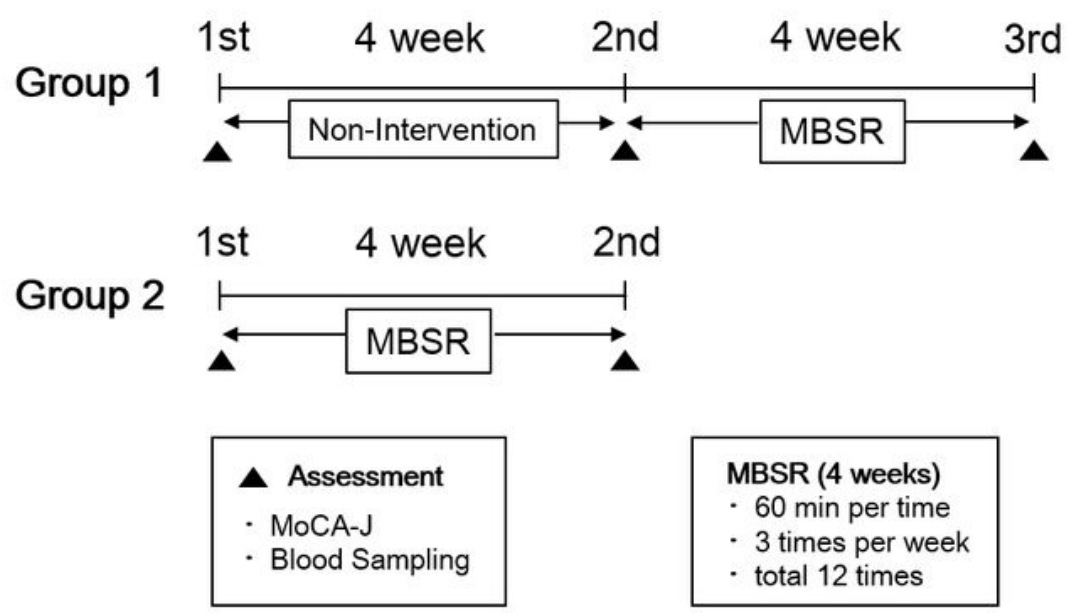

b

Group 1

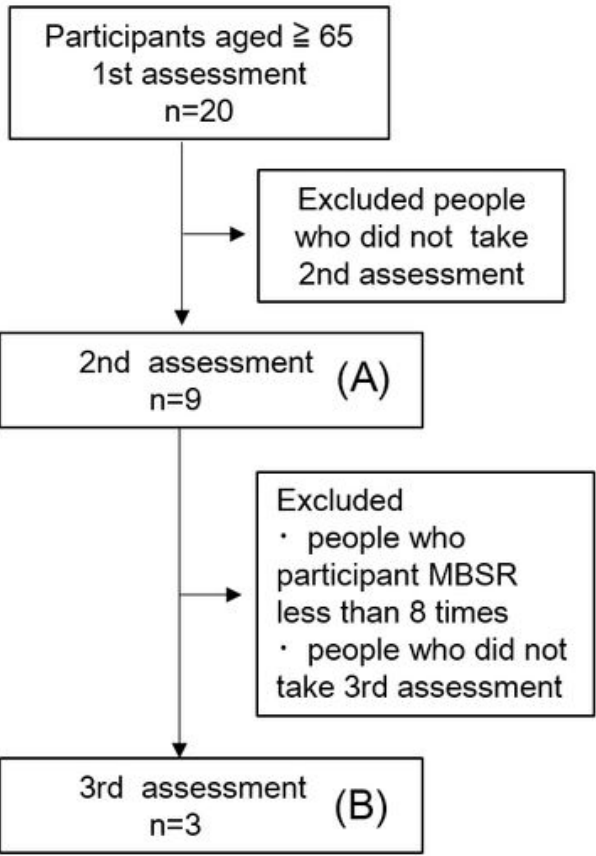

\section{Group 2}

Participants aged $\geqq 65$

1st assessment $\mathrm{n}=25$

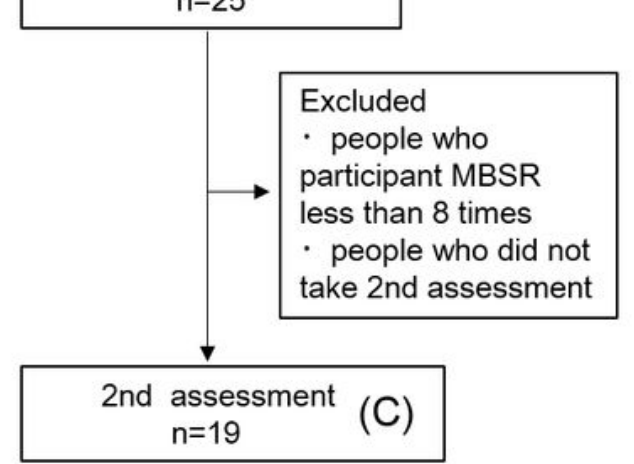

Non-MBSR group : $(A)(n=9)$

MBSR group : $(B)+(C)(n=22)$

\section{Figure 1}

Flow chart of the study design. (a) In Group 1, no intervention was performed for 4 weeks, and then the MBSR intervention was conducted for 4 weeks. In Group 2, only the MBSR intervention was performed for 4 weeks. During the intervention, three MBSR sessions were performed per week for a total of 12 sessions within 4 weeks. Each MBSR session was performed for $60 \mathrm{~min}$. Before and after the intervention, the MoCA-J and blood sampling were conducted. (b) A total of 45 older adults aged over 65 years 
participated in this study (Group 1: $n=20$, Group 2: $n=25$ ). We excluded the data of participants who performed the MBSR program fewer than eight times or who did not undergo the second or third assessment. We analyzed the data of the 1st and 2nd assessments of Group 1 as the non-MBSR group $(n=9)$. We also analyzed the data of the $2 n d$ and 3rd assessments of Group $1(n=3)$ and the data of the 1 st and 2nd assessments of Group 2 as the MBSR group $(n=19)$. The data of all 22 participants were evaluated as the MBSR group.

\section{Figure 2}

a

Total MoCA-J Score

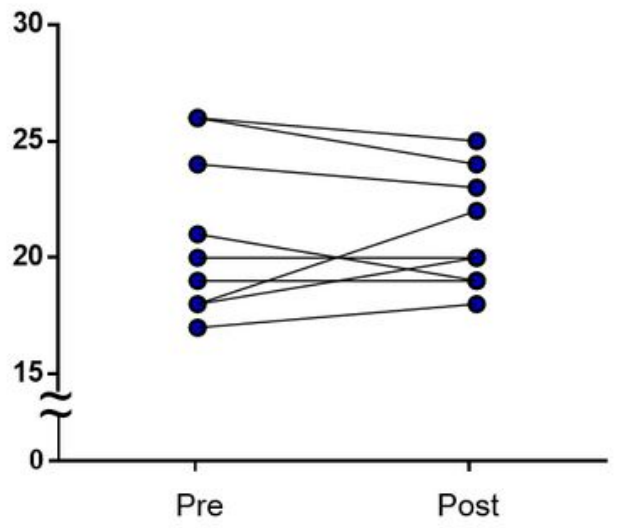

b

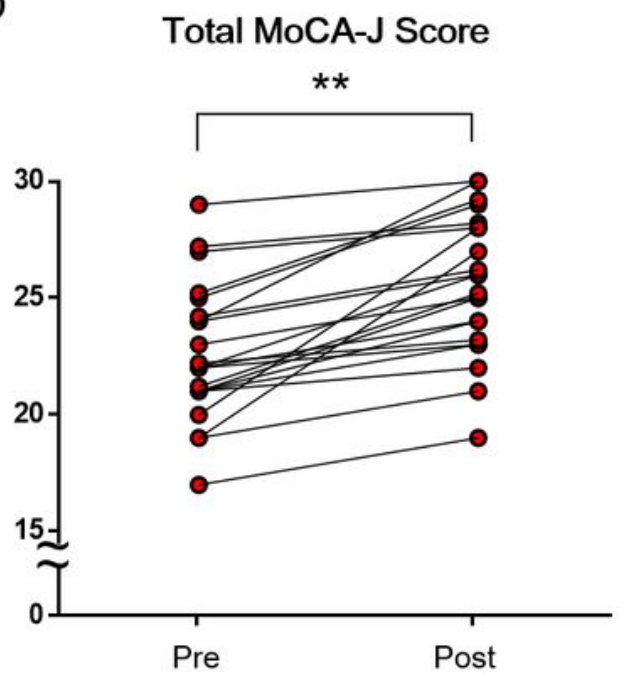


Change in the total score for the MoCA-J in the non-MBSR and MBSR groups. The total score for the MoCA-J did not change in the non-MBSR group (a), but the score was significantly increased in the MBSR group after the intervention (b). ${ }^{*} P<0.01$, paired t-test. non-MBSR group $(n=9)$, MBSR group $(n=22)$.

\section{Figure 3}

a

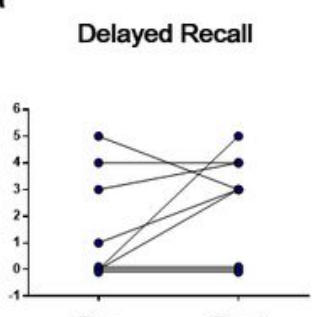

Pre Post
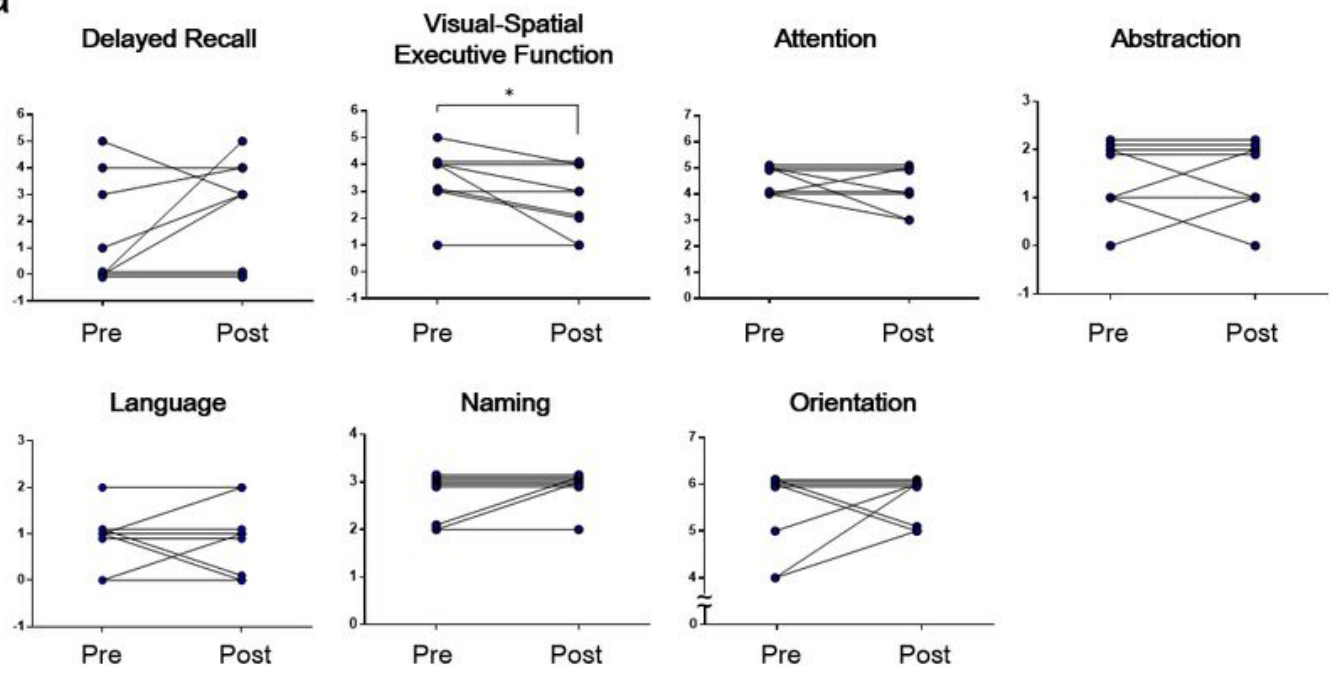

b
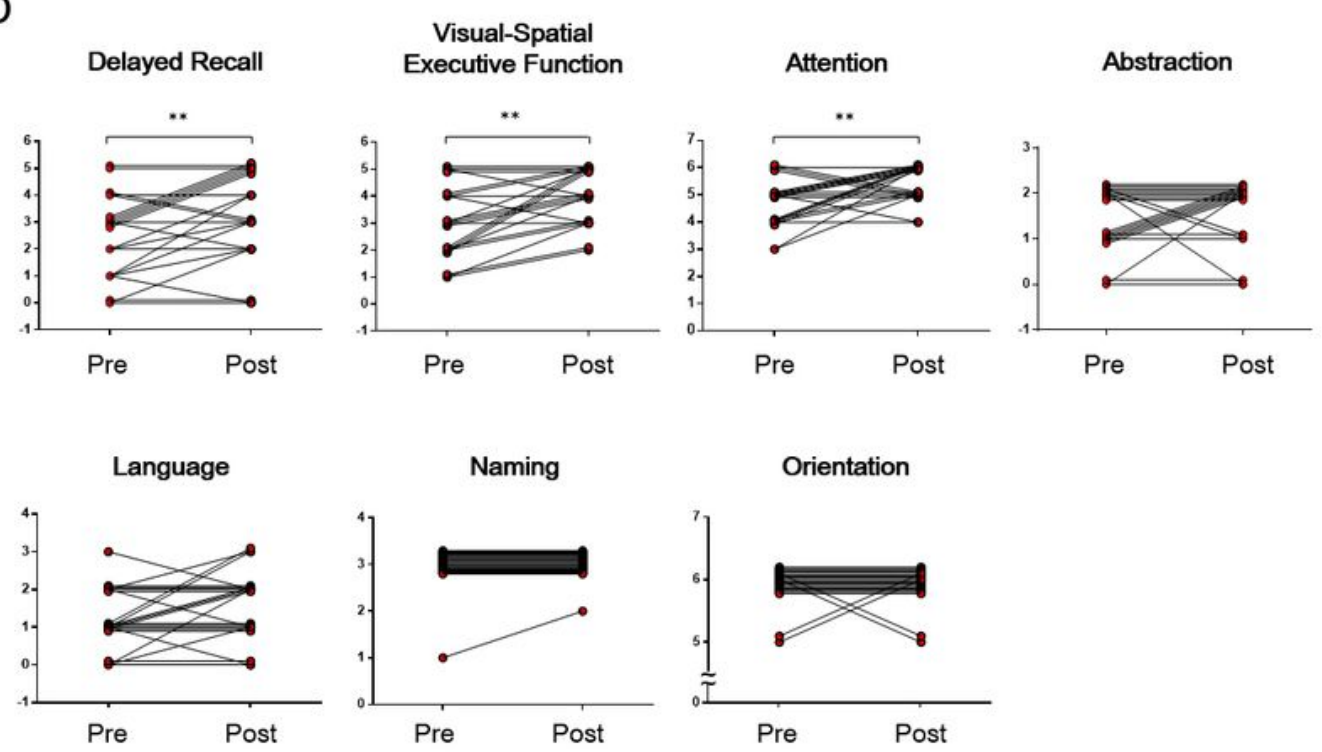

\section{Figure 3}

Change in the score for the seven domains of the MoCA-J in the non-MBSR and MBSR groups. The changes in the score for the seven domains of the MoCA-J, including delayed recall, 
visuospatial/executive function, attention, abstraction, language, naming, and orientation, were assessed in the non-MBSR (a) and MBSR groups (b). In the non-MBSR group, the score for visuospatial/executive function was significantly decreased in the non-MBSR group after no intervention. In the MBSR group, the scores of delayed recall, visuospatial/executive function, and attention were significantly increased after the intervention. ${ }^{*} P<0.05,{ }^{*} P<0.01$, paired t-test. non-MBSR group $(n=9)$, MBSR group $(n=22)$.

\section{Figure 4}

a

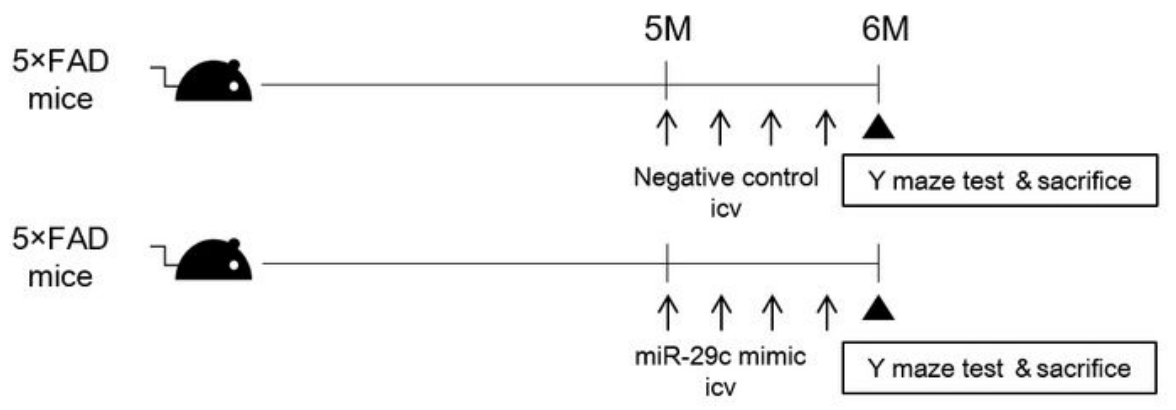

b

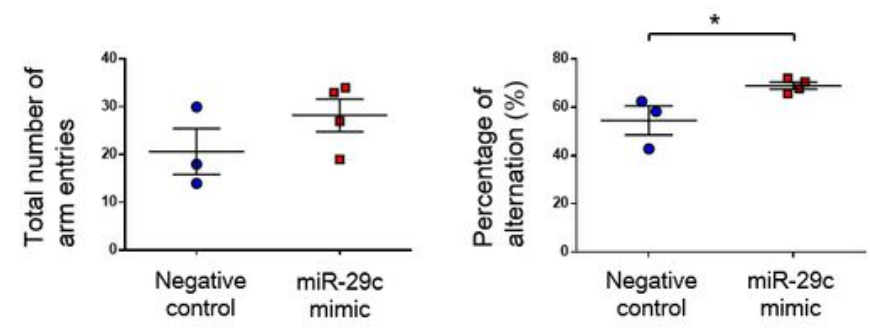

C

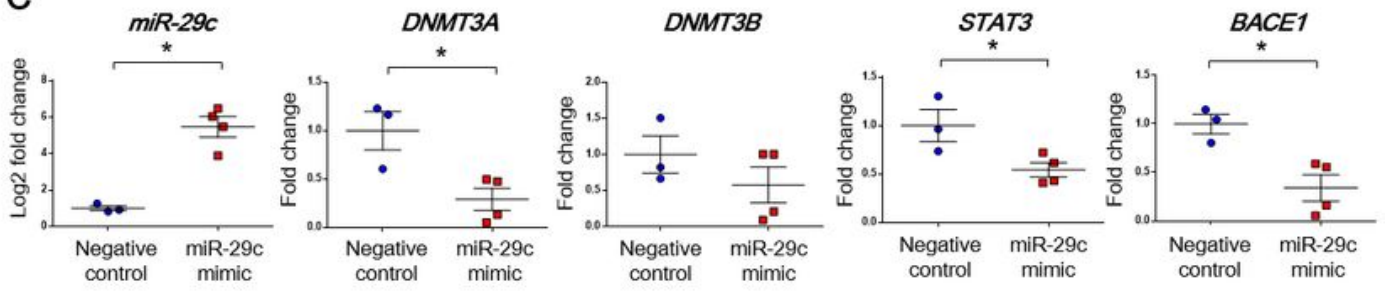

d
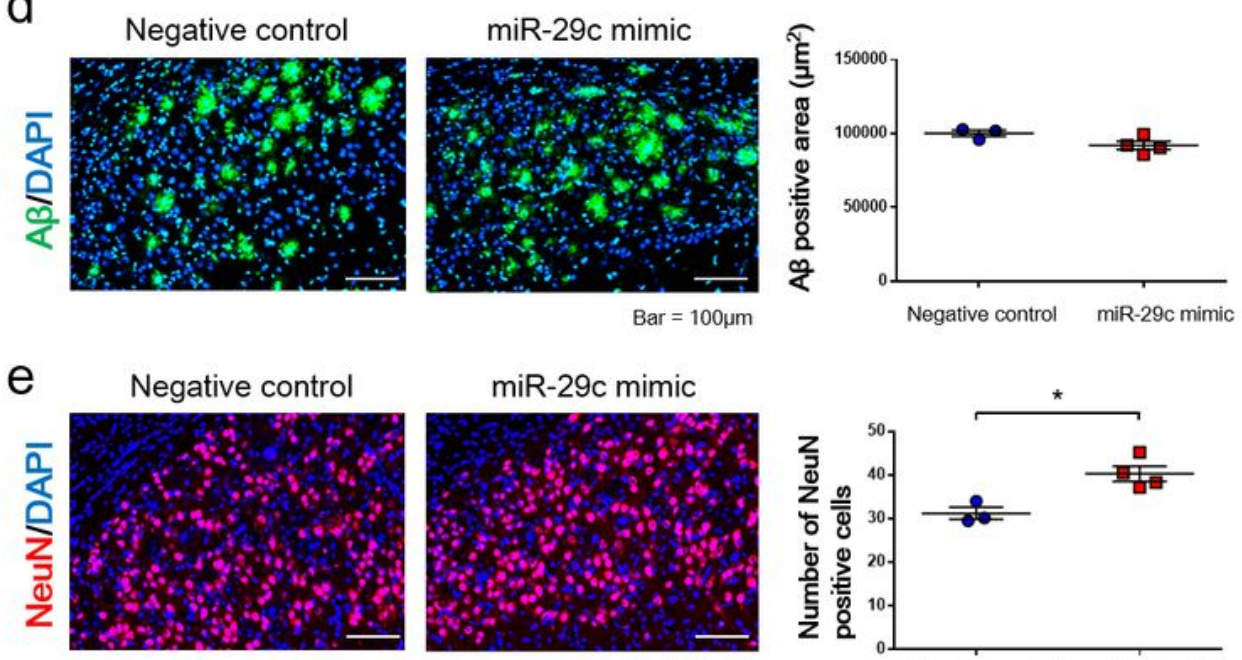

miR-29c mimic
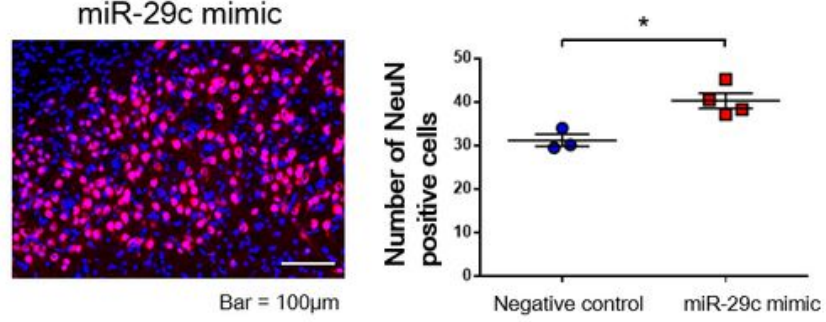

Figure 4 
Effect of intracerebroventricular injection of the miR-29c mimic into 5xFAD mice. (a) Experimental protocol. Negative control miRNA (140 pmol) or miR-29c mimic (140 pmol) was injected intracerebroventricularly into 5-month-old 5xFAD mice four times at 1-week intervals. At 1 week after the last injection, $Y$ maze tests were conducted, and mice were sacrificed at 6 months old. (b) The result of $Y$ maze tests. Total numbers of arm entries and the percentages of alternations are shown. Values are the means \pm SEM. $* P<0.05$, unpaired t-test. Negative control group $(n=3)$, miR-29c mimic group $(n=4)$. (c) The expression of miR-29c, DNMT3A, DNMT3B, STAT3, and BACE1. Values are the means \pm SEM. *P < 0.05 , unpaired t-test. Negative control group $(n=3)$, miR-29c mimic group $(n=4)$. (d) The Aß-positive area. Values are the means \pm SEM. Negative control group $(n=3)$, miR-29c mimic group $(n=4)$. (e) The number of NeuN-positive cells. Values are the means \pm SEM. ${ }^{*} P<0.05$, unpaired t-test. Negative control group $(n=3)$, miR-29c mimic group $(n=4)$.

\section{Supplementary Files}

This is a list of supplementary files associated with this preprint. Click to download.

- Supplementarylnformation.pdf

- Table1.jpg

- Table2.jpg

- Table3.jpg 